\title{
The Motion Simulation System of Steel Cutting Manipulator Xianghong $\mathrm{Xu}$

\author{
Shazhou Professional Institute of Technology, Zhangjiagang 215600, China \\ email: xxh602@126.com
}

\begin{abstract}
Keywords: MATLAB; the robot motion simulation system; Virtual Lab; Steel cutting manipulator; Plan manipulator trajectories
\end{abstract}

\begin{abstract}
Using the Visual Studio and MATLAB to carry on secondary development in Pro/E and complete the robot motion simulation system. Robotics Toolbox is used to establish the dynamic model of steel cutting manipulator ; Through the joint development of the Visual Studio and the Pro/E, we can import robot model through the main interface, open the robot work space, and enter the MATLAB simulation interface in Pro/E. On the basis of the robot kinematics, the motion simulation system is made out to verify the rationality of the design of the steel cutting robot, and take flange beam for instance to plan manipulator trajectories. Finally, the paper puts forward some suggestions about the further improvement and refinement on the CNC steel cutting at the actual work.
\end{abstract}

\section{Introduction}

The cutting process of steel into the cutting path and cutting characteristics, cutting path is straight line, circle, ellipse, ellipse arc primitives, cutting features a variety of information feature primitives (type of groove, groove angle) [1]. For H steel, I-beam, channel steel and angle steel, these four kinds of steel have non rotary characteristics and multi plane structure, so they are difficult to cut. According to the type of steel clamping way, need to consider the rotation of [2] type steel cutting device for cutting table and cutting torch. The complete process of steel cutting steel cutting graphics requirements, and to ensure the torch and workpiece without interference, avoid touching the torch and the accident [3]. The following is an example of i-beam.

\section{Theoretical Analysis}

\section{Cutting Path Process}

The cutting space of I-beam is complex and requires the superposition of $\mathrm{X}, \mathrm{Y}$ and $\mathrm{Z}$ axes to cut the [4]. Steel cutting torch pose as shown in Figure 1, cut by the numbers in the figure of the process sequence.

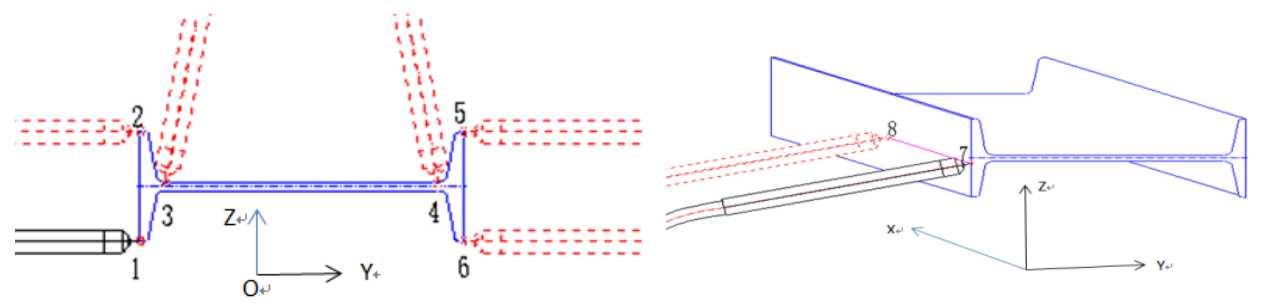

Fig. 1. Steel cutting torch pose

Groove cutting is the cutting angle of rotation into the groove. In Figure 2, when the steel cutting machine hand cutting I-beam $\mathrm{Y}$ groove, torch pose for the $\mathrm{X}$ axis direction of anti clockwise 60 degrees, cut from point a to point B. H type steel cutting process and I-beam is similar; do not need to drop the gun channel; equal angle is 90 degrees over cutting edges, the initial angle is 45 de grees; unequal angle according to the calculated length of the initial angle of torch[5-6]. The cutting 
process is similar to I-beam, no more details. Steel cutting manipulator device is composed of a single torch manipulator and a cutting device. The design scheme of the steel cutting manipulator is shown in figure 3. The manipulator 1 bar, 2 bar, 2 through 1 linkage joint rotation in $\mathrm{Y}, \mathrm{Z}$ plane, $\mathrm{X}$ to move straight and $\mathrm{YZ}$ plane rotating cutting device working table, realize the change of space position of cutting torch; joint 3 and joint 4 round 2 rod to rotate the cutting torch do swing up and down, achieve the required cutting groove the rod 4 is cutting angle. Figure 2 single torch manipulator design and cutting device for working table Steel cutting manipulator device needs to achieve the type commonly used steel (steel, steel, H steel, I-beam) cutting lines and oblique lines and groove space, cutting section is complex, the single torch manipulator and cutting device table form can meet the demand of steel cutting. Analysis of mechanical hand cutting path, through the method of solving planar two rod joint, the manipulator workspace torch as shown in figure 3 .

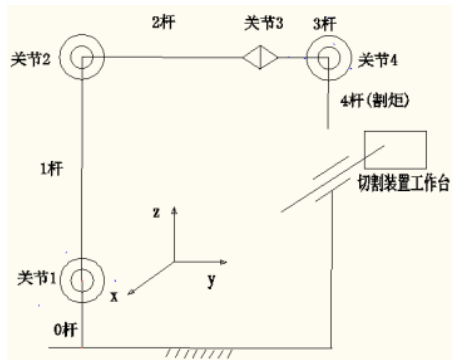

Fig.2. Single torch manipulator

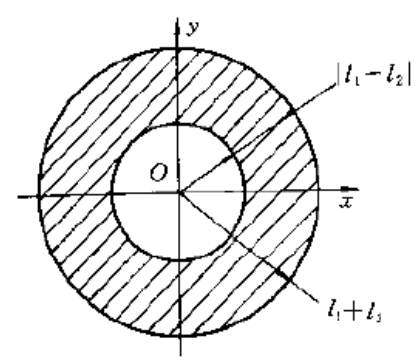

Fig.3. The manipulator workspace torch

\section{Cutting Path Simulation of Steel Cutting Manipulator}

With the help of MATLAB platform, the three-dimensional model of steel cutting manipulator is constructed by using Robotics Toolbox, and taking the I-beam as an example, the trajectory simulation based on joint space and Cartesian space is carried out. Robotics steel cutting manipulator. A three-dimensional model of a steel cutting manipulator is constructed in Robotics Toolbox, as shown in Figure 4. Simulation of manipulator with 2.2 cutting paths. Taking I-beam 20A $(200 * 100 * 7)$ as an example, the cutting path simulation of steel manipulator is carried out by MATLAB. In MATLAB, the set of manipulator with 1 reference coordinates $x, y, Z$, mechanical hand torch ( 3 bar) pose for $\mathrm{N}, \mathrm{O}$, a. The I-beam is clamped at the cutting table and moves forward along the $\mathrm{Z}$ axis. Each section of the section is moved in a length of section steel. The machine tool rotates along the XY plane to change the position of the steel section. The coordinates of the origin coordinate system are $(0,0,0)$, and the coordinate of the rotating center of the worktable is $(1160690,0)$. Through the analysis of position between the torch and the origin of the work, follow the shortest path principle, technology position cutting 20A I-beam, the location of the corresponding position. When the torch is vertically placed in Table 1 at position 1 , through the analysis of relationship between the end position of the cutting torch and the origin of the work attitude of T1: Get theta $1=1.5708$, theta $2=-1.5708$, theta $3=-1.5708$. Similarly, the steel cutting robot can move to the table 3 , position 2 to 10 when the joint turns theta.

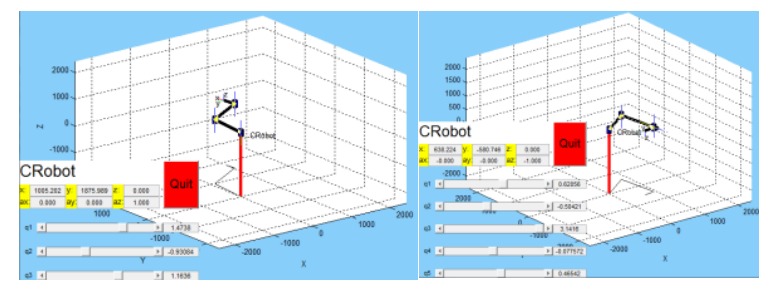

Fig. 4 Animation demonstration of steel cutting manipulator

Through the Robotics Toolbox's plot function, the movement process of the three-dimensional animation form of the steel cutting manipulator is obtained. MATLAB Robotics Toolbox provides the Trajan function (five polynomial interpolation function) can achieve joint space planning [5]. 
Assuming the steel cutting manipulator from position 1 uniform motion in 10 seconds 2 , according to table 2 for the joint angle, namely q1=[1.5708 -1.5708 1.5708] and q2=[1.4706 -1.4661 -1.5753$]$, through MATLAB programming, can get the manipulator from position 1 to 2 when the movement of each joint angle theta, speed and acceleration of q/ V A and displacement of the manipulator end cutting torch.

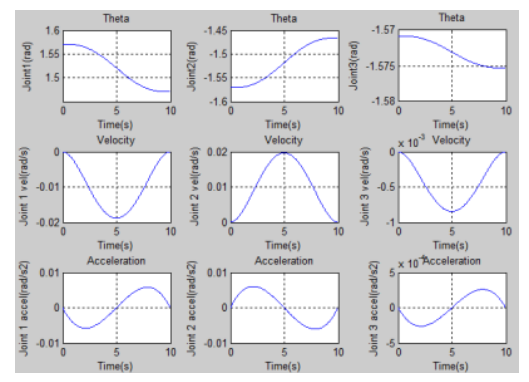

Fig. $5 \mathrm{Q}, \mathrm{V}$ and a of manipulator cutting path

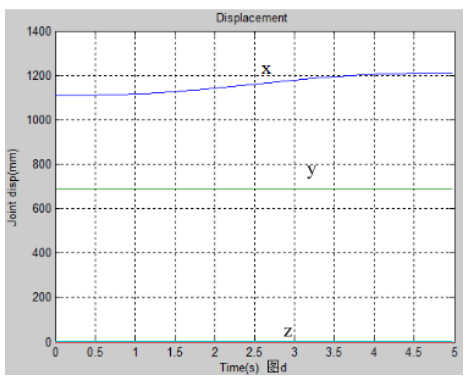

Fig.6 The cutting torch cutting path

Fig.5 representative steel cutting manipulator when cutting I-beam from Figure 6 position movement joint angle, velocity and acceleration, from the diagram that the manipulator joint motion is normal, smooth motion, no dislocation link conflict; figure 6 for cutting displacement curve of the manipulator, from the diagram that torch in the YZ plane along the $\mathrm{X}$ axis from the 1110 to the 1210 position, end joint displacement of I-beam technology requirements. Similarly, the motion parameters of position 2 to 10 can be obtained. In addition, through programming, you can see motion parameters, images and robot hand motion animation.

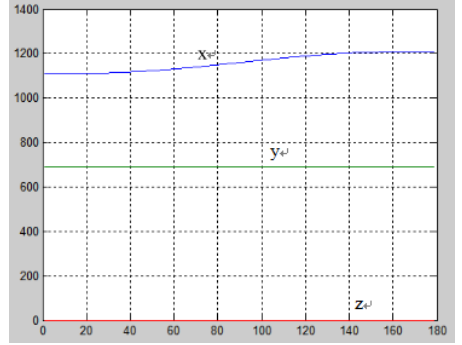

Fig.7 Torch displacement manipulator

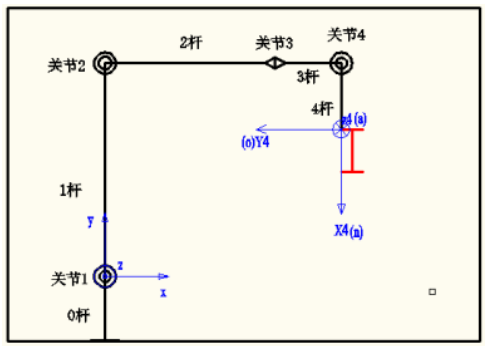

Fig. 8 Cutting machine manipulator

Cartesian programming is achieved by using the ctraj function (linear interpolation) in MATLAB Robotics Toolbox [6]. Displacement type steel cutting torch at the end of the arm in Figure 10, the manipulator torch in the $\mathrm{YZ}$ plane along the $\mathrm{X}$ axis from the 1110 to the 1210 position, end joint displacement of I-beam technology requirements. Simulation of manipulator with 2.3 cutting grooves. In this paper, the cutting groove of $\mathrm{Y}(7 \mathrm{~mm})$ MATLAB groove is analyzed. The manipulator scheme for cutting the groove is shown in Figure 8. The position and position of the $Y$ groove of the cut I-beam. The torch is vertically arranged in Figure 11 at position a, analysis of the relationship between the torch and the work origin position, mechanical hand joint angles. Similarly, we can know the movement process of the three dimensional animation form of the steel cutting manipulator. The kinematic parameters based on the joint space in the cutting groove of the steel cutting manipulator. This is the manipulator's joint angle, speed and acceleration curve from position a to $\mathrm{B}$. The figure shows that the joint motion of the manipulator is smooth, and the linkage has no dislocation phenomenon, and the design of the linkage parameters is reasonable. The based on the displacement of the manipulator joint space cutting torch cutting groove The displacement of the manipulator end of the torch, torch from the figure in the $\mathrm{YZ}$ plane along the $\mathrm{X}$ axis from 1110 to 1010 at the end of the torch moving position, displacement of I-beam technology requirements. The steel can be obtained at the end of the torch displacement Cartesian space manipulator based on cutting cutting groove. Based on the torch displacement manipulator Descartes coordinate space cutting groove That the manipulator of cutting in the $\mathrm{YZ}$ plane along the $\mathrm{X}$ axis from 1110 to 1010 at the end of the torch moving position, displacement of I-beam technology requirements. 


\section{Motion Simulation System Interface}

In the Pro/E4.0 and Visual Studio 2005 with two development, completed the manipulator movement simulation system, the main interface and manipulator workspace display interface design. Through programming and debugging, through the master. Interface import manipulator model, open manipulator, workspace display interface and MATLAB simulation system. The Pro/E interface menu and dialog box are shown in figures 9. Motion simulation" button can be achieved by opening MATLAB, and by running the edited *.m file, open the cutting steel Manipulator Simulation interface.
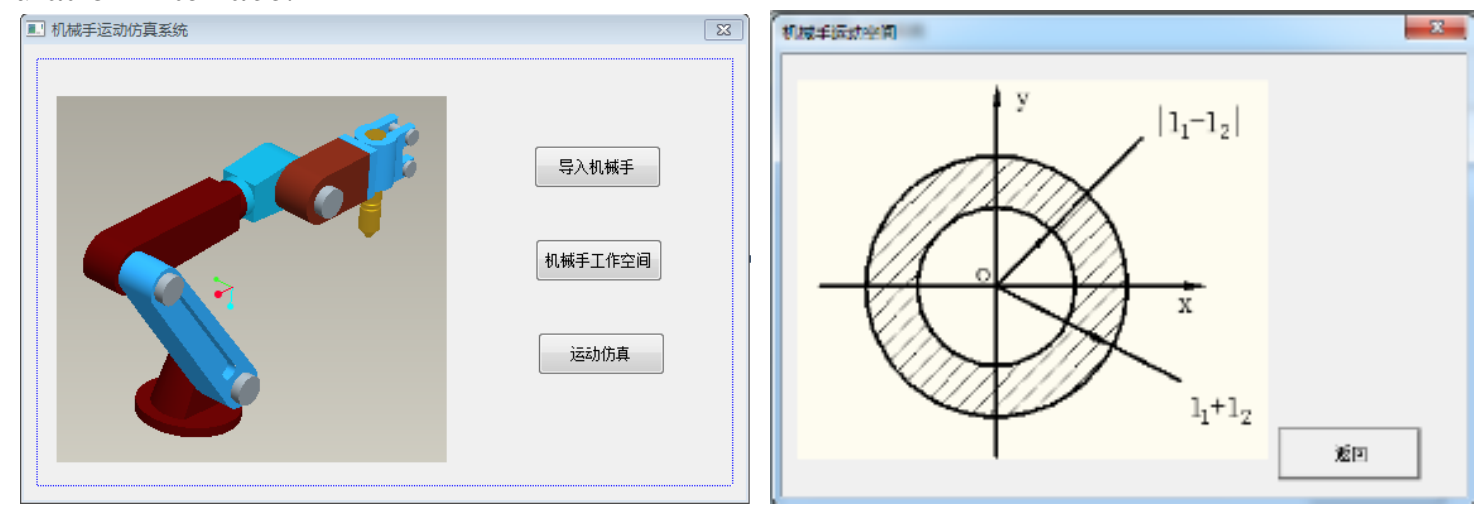

Fig.9 Main interface

\section{Reference}

[1] Liu Hongwei, Xue Dangqin. Five DOF robot forward and inverse kinematics simulation research [J]. new technology, new technology, 2009,02:64-67.

[2], Zhang Huan. Structural design, kinematics analysis and Simulation of robot with six degrees of freedom [D]. At University, 2004:18-20. Kinematics analysis and Simulation.

[3] Zhang Hongtao 3-3UPS1S parallel robot [D]. Jiangnan University Learn, 2008:99-102.

[4] Xie Bin, Cai Zixing. Based on the MATLAB Robotics Toolbox robot imitation Real experimental teaching, [J]., computer education, 2010, No.12719:140-143.

[5], Dong Huiying, Liang Shuang. Kinematics simulation of PUMA560 robot based on MATLAB [J]. Wireless Interconnect Technology, 2014, No.4505:179-180.

[6], Wang Chieh. Six DOF robot body design and trajectory planning and virtual simulation [D]. HeFei University of Technology, 2015:76. 